\title{
Empatía en Autismo: concepto y medición ${ }^{* *}$
}

\author{
Empathy in Autism: Conception and Measurement
}

Empatia no Autismo: Conceito e mediação

\footnotetext{
* Asistente de investigación Colciencias. Psicóloga, magíster en formación (en la maestría de estudios sociales y políticos modalidad investigación). a) Investigación en cognición social, b) investigación en bases cerebrales, investigación e intervención en autismo, 3) investigación de bases cerebrales y conducta social en sujetos privados/negligencia a edad temprana. Primer puesto en el I concurso de conocimiento en neurociencia de la Universidad Tecnológica de Pereira (2014), proyecto de grado con mención honorífica. ORCID: http://orcid.org/oooo-0002-7259-7163. Correo electrónico: vivianariveraortega@gmail.com

** Este artículo de investigación hace parte de los compromisos de formación posgradual de la autora adquiridos en el proyecto 211774454967 de Colciencias. Artículo de investigación recibido el 19-01-2018 y aceptado 06-04-2018.
} 


\section{Cómo citar}

Rivera Ortega, V. (2018). Empatía en Autismo: concepto y medición. CS, (25), 191-211.

DOI: http://dx.doi.org/10.18046/recs.i25.2707 
Esta revisión analiza la aproximación conceptual y la medición de la empatía en los trastornos del espectro autista. La empatía es un constructo que tiene diversas aproximaciones y abordajes en las ciencias cognitivas. Se puede determinar que existen diversas concepciones de empatía. Estudios muestran una dicotomía en el procesamiento de la empatía en sujetos con autismo, sugiriendo un déficit de empatía en el funcionamiento cognitivo, pero no en el afectivo. En el estudio de estas áreas se encuentra una tendencia en el uso de autorreportes, cuestionarios y tareas de teoría de la mente. De otro lado, las mediciones cerebrales han mostrado una activación atípica en la corteza prefrontal derecha, la unión temporoparietal, la corteza insular y el giro fusiforme.

PALABRAS CLAVE:

Empatía, autismo, neuropsicología, cognición, afecto, áreas cerebrales

This review analyzes the conceptual approach and measurement of empathy in the disorders on the autism spectrum. Empathy is a construct encompassing a variety of approaches in the cognitive sciences. In can be established that a diversity of conceptions of empathy exist. Studies show a dichotomy in the processing of empathy in subjects with autism, suggesting a deficit of empathy in cognitive functioning but not in affective functioning. In the study of these areas, there is a tendency to employ self-reports, questionnaires, and theory of mind tasks. however, brain measurements have shown atypical activations of the right prefrontal cortex, the temporoparietal junction, the insular cortex and the fusiform gyrus.

\section{KEYWORDS:}

Empathy, Autism, neuropsychology, cognition, affect, brain areas 
Esta revisão analisa a aproximação conceitual e a mediação da empatia nos transtornos do espectro autista. A empatia é um construto que tem diversas aproximações e abordagens nas ciências cognitivas. Pode-se determinar que existem diversas concepções e empatia. Estudos mostram uma dicotomia no processamento da empatia em sujeitos com autismo, sugerindo um déficit de empatia com o funcionamento cognitivo, mas não no afetivo. No estudo destas áreas encontra-se uma tendência no uso de auto reportes, questionários e tarefas de teoria da mente. Por outro lado, as mediações cerebrais têm mostrado uma ativação não típica na no córtex pré-frontal direito, uma união temporoparietal, o córtex insular e o giro fusiforme.

\section{PALAVRAS CHAVE:}

Empatia, autismo, neuropsicológica, cognição, afeto, áreas cerebrais 


\section{Introducción}

Desde que Titchener (1909) definió la empatía como la capacidad de «sentirse dentro de», el concepto ha sido objeto de numerosas discusiones teóricas que han variado de acuerdo con los enfoques que la han sustentado (Fernández-Pinto, López-Pérez y Márquez, 2008). De esta forma, se encuentran posturas teóricas que plantean la empatía como una respuesta emocional y comportamental a los estados mentales y afectivos, y a los pensamientos de un observador hacia un observado, implicando reacciones de filiación (Albiero, Matricardi, Speltri y Toso, 2009; Decety y Lamm, 2006; Pelligra, 2011; Stocks, Lishner, Waits y Downum, 2011). En una línea similar, se ha sostenido que la empatía es la capacidad para comprender, anticipar y reaccionar a los estados mentales de los otros, pero con distinción plena entre lo propio y lo ajeno; es decir, saber que aquello que se está experimentando es producido por un externo (Clark, 2010; Decety y Michalska, 2010; Oliveira-Silva y Gonçalves, 2011).

Algunas formulaciones teóricas sustentan una división entre los procesos cognitivos y afectivos, definiendo la empatía como una habilidad para la comprensión cognitiva y afectiva de los estados mentales de los otros, con capacidad para tomar perspectiva y asumir una reacción emocional (Barnett y Mann, 2013; Feshbach, 1975; Singer y Lamm, 2009). Otros autores proponen que la empatía tiene que ver con la preocupación subyacente al sufrimiento o al peligro en el que se encuentra otra persona, provocando sentimientos como la compasión y la ternura (Geer, Estupinan y Manguno-Mire, 200o) o que es una simple capacidad de imitación (Goldman, 1993), incluso como una forma básica de intencionalidad (Zahavi, 2008). También, el concepto ha sido comprendido como un proceso ligado estrechamente al funcionamiento de las neuronas espejo (Cattaneo y Rizzolatti, 2009).

En este sentido, es posible plantear que la empatía ha sido abordada y entendida como un proceso, una habilidad o una respuesta comportamental. En los estudios de psicología experimental y neurociencia cognitiva tiende a entenderse la empatía como una característica de la mente que no solo permite entablar una relación con un otro, sino que además posibilita la comprensión de los estados emocionales propios y ajenos (Bird y Viding, 2014; Sucksmith, Allison, Baron-Cohen, Chakrabarti y Hoekstra, 2013). Supone también la capacidad para entender y responder a experiencias emocionales propias de los otros (Decety y Jackson, 2006).

El estudio de la empatía ha abordado por separado sus componentes afectivos y cognitivos. Respecto al procesamiento afectivo, se ha definido como un afecto compartido o un sentimiento vicario (Lockwood, 2016), mientras que el procesamiento cognitivo se ha entendido como la capacidad de comprender y adoptar la perspectiva y los pensamientos de los otros (Fernández-Pinto et al., 2008). Aunque existen 
diferentes postulados teóricos, la empatía constituye uno de los dominios básicos para la interacción social, puesto que posibilita la comprensión de otras personas y facilita el establecimiento de relaciones sociales (Lockwood, 2016). Partiendo de estas concepciones, en las últimas décadas la empatía se ha estudiado empíricamente en patologías neuropsiquiátricas como los trastornos del espectro autista (TEA), este interés ha surgido debido a que el autismo se caracteriza por el déficit en la comunicación (Bos y Stokes, 2018) y las habilidades sociales, llevando a la ineficacia en el establecimiento de relaciones sociales (Ruggieri, 2013), convirtiéndose en un modelo clínico ideal para estudiar diversos dominios de la cognición social.

Los estudios empíricos han utilizado diversos instrumentos, entre los cuales es común denominador el uso de autorreportes o cuestionarios (Bird et al., 2010; Hagenmuller, Roessler, Wittwer y Haker, 2014; Mathersul, McDonald y Rushby, 2013; Sucksmith et al., 2013) y tareas de rendimiento (Koehne, Hatri, Cacioppo y Dziobek, 2016; Schulte-Rüther et al., 2014) que han esclarecido los déficits de empatía en el autismo (Aan het Rot y Hogenelst, 2014). Así mismo, cuestionarios utilizados para la medición de empatía han mostrado que los sujetos con autismo presentan déficits más prominentes en el componente cognitivo de la empatía (Dadds et al., 20o8; Pouw, Rieffe, Oosterveld, Huskens y Stockmann, 2013).

Lo anterior está estrechamente relacionado con estudios que muestran que en teoría de la mente (ToM, del inglés theory of mind $)^{1}$ los autistas presentan una marcada dificultad para comprender los estados emocionales, lo cual puede explicar las dificultades encontradas en tareas de reconocimiento utilizadas en la medición de la empatía cognitiva (Mazza et al., 2014), contradiciendo así algunos hallazgos que muestran que la ToM no se encuentra relacionada directamente con la empatía (Peterson, 2014). De igual forma, se encuentran estudios que sugieren que no solo la empatía cognitiva se altera en autismo, también la interacción con aspectos afectivos y habilidades sociales (Grove, Baillie, Allison, Baron-Cohen y Hoekstra, 2014).

Con relación a la investigación de las bases cerebrales de la empatía en autismo, los estudios han utilizado técnicas de neuroimagen como la fMRI (del inglés: functional magnetic resonance imaging). Estos trabajos muestran una tendencia en la reducción de la activación en la corteza prefrontal dorsomedial, un procesamiento atípico en el área posterior del surco temporal superior, la amígdala y la corteza cingulada anterior (Ruggieri, 2013). De igual forma, se ha expuesto que la unión temporoparietal tiene un importante rol en la empatía, puesto que permite discriminar las creencias e intenciones de las otras personas en el funcionamiento de la teoría de

1. El concepto suele entenderse como la capacidad de un sujeto para comprender cómo los estados mentales internos, incluyendo pensamientos y sentimientos, que forman el comportamiento humano, son distintos de la experiencia externa (Peterson, 2014). 
la mente (Fan, Chen, Chen, Decety y Cheng, 2014; Hoffmann, Koehne, Steinbeis, Dziobek y Singer, 2016; Tousignant, Eugène y Jackson, 2016); así como la corteza orbitofrontal se encarga de procesar y regular las emociones y la conducta (Lázaro y Solís, 2008). Las cortezas occipitales y límbicas también se han visto involucradas en mayor actividad en estudios que evalúan conductas empáticas (Geday, Gjedde, Boldsen y Kupers, 2003; Moya-Albiol, Herrero y Bernal, 2010). Lo anterior permite inferir que la empatía es un proceso determinante en la interacción social, que parece estar afectado en autismo. Es por esto que esta revisión analiza los hallazgos y métodos de medición que se han utilizado en recientes estudios que abordan la empatía en autismo.

\section{Mediciones conductuales de la empatía en autismo}

\section{Disociación de la empatía}

El abordaje experimental de la empatía ha conducido a esta premisa, existen dos procesamientos: a) el cognitivo, que se ha entendido como la habilidad para reconocer y comprender los sentimientos de los otros, involucrando una relación cercana con la teoría de la mente (Blair, 2005); y b) el afectivo, basado en la reacción emocional de un observador que percibe a otro experimentando o a punto de experimentar una emoción (Stotland, 1969), también llamado recientemente como resonancia afectiva (Decety y Meyer, 2008) o contagio emocional (Shamay-Tsoory, Tomer, Yaniv y Aharon-Peretz, 2002).

Derivado de estas posturas, Dziobek et al. (2008) plantearon un estudio para analizar la disociación entre la empatía cognitiva y afectiva en adultos con Síndrome de Asperger (SA). Utilizaron el Multifaceted Empathy Test - MET (Dziobek et al., 2006), el cual consiste en 23 pares de fotografías (estímulos) que presentan personas y contextos sobre los que deben decidir un estado mental; paralelamente, se empleó el Self Assessment Manikin (Lang, Bradley y Cuthbert, 1997) para medir el grado de excitación de cada participante frente al estímulo. Para medir la empatía utilizaron Interpersonal Reactivity Index - IRI (Davis, 1980). Los resultados muestran que sujetos con SA obtuvieron, tanto en el MET como en el IRI, un puntaje significativamente más bajo que el grupo control en la empatía cognitiva. En relación a la empatía afectiva, no se hallaron diferencias significativas. Estos resultados sugieren que los individuos con SA presentan un déficit en el procesamiento propio de la empatía cognitiva, contrario con los mecanismos de la empatía afectiva, la cual se muestra conservada. 
Por su parte, Aan het Rot and Hogenelst (2014) estudiaron la influencia de los rasgos autistas y la empatía afectiva sobre la precisión empática en adultos jóvenes autistas. Para ello, evaluaron la empatía afectiva con el Balanced Emotional Empathy Scale - BEES (Mehrabian, 1996). En esta investigación también se evaluó la precisión empática, implicando la calificación de los sentimientos de los otros y el relato verbal de eventos emocionales autobiográficos por medio de videos (basados en Zaki, 2008). Los resultados muestran que no hubo diferencias significativas en precisión empática entre hombres y mujeres. Se encontró una asociación negativa entre la precisión empática y el autismo entre sujetos con bajo autorreporte de empatía afectiva, pero no en los de empatía cognitiva, corroborando estudios previos (Mathersul et al., 2013; Shamay-Tsoory et al., 2002). Así mismo, cabe resaltar que las mujeres obtuvieron mejores puntuaciones en la precisión empática, indicando que el procesamiento de los estímulos puede estar atravesado por el sexo del observador.

No todos los estudios muestran disociación entre los mecanismos de la empatía entre sujetos autistas y sujetos con desarrollo típico. Prueba de ello es un estudio realizado por Cook, Brewer, Shah y Bird (2013), cuyos resultados no arrojaron diferencias significativas entre grupos, en cuanto a la capacidad de interpretar los estados emocionales de los otros, pero sí una asociación negativa entre los participantes en los resultados de las tareas que median aleximia. Por su parte, Pasalich, Dadds y Hawes (2014) utilizaron The Griffith Empathy Measure (Dadds et al., 2008) para evaluar la empatía en niños autistas y muestran que las deficiencias más significativas en autistas se relacionan con niveles más bajos en empatía cognitiva. Lo anterior es inconsistente con estudios que no han encontrado déficits respecto a los dominios antes descritos (Jones, Happé, Gilbert, Burnett, y Viding, 2010; Schwenck et al., 2012).

De acuerdo con los estudios revisados, es posible plantear que las investigaciones realizadas sobre la empatía en autistas sugieren que los procesos empáticos siguen dos líneas: por un lado, se encuentra que existe un posible déficit en el reconocimiento de los estados emocionales y en la capacidad de tomar la perspectiva del otro; pero, por otra parte, se encuentran comportamientos de correspondencia emocional acerca de los sucesos o eventos con carga emocional positiva. Esto se evidencia en resultados donde la empatía afectiva se ha mostrado conservada y la empatía cognitiva disminuida. Los estudios han mostrado que, cuando existen puntajes altos en tareas que evalúan la empatía cognitiva, se muestra una asociación positiva entre la precisión empática y los rasgos autistas, contrario cuando los puntajes de empatía afectiva son bajos. 


\section{Empatía y teoría de la mente}

Un reciente estudio que analiza conjuntamente ToM y empatía en adolescentes (Mazza et al., 2014), utilizó el Theory of Mind Task (Happé, 1994) y la versión revisada del Reading the Mind in the Eyes Test (Baron-Cohen, Wheelwright, Skinner, Martin y Clubley, 2001) para medir ToM, y en él se mostró que los sujetos con autismo presentan un déficit en todas las mediciones de mentalización, sugiriendo que son incapaces de interpretar y entender los estados emocionales de los otros. En la medición de compartir experiencias, los adolescentes con autismo fueron capaces de empatizar con la experiencia afectiva de otras personas cuando ellos expresaban emociones positivas, pero no cuando la emoción era negativa; sin embargo, cuando se mostraba la región de los ojos, no eran capaces de reconocer las emociones. De acuerdo a esto, el estudio sugiere que el déficit en la dimensión de la empatía podría deberse al marcado déficit en la habilidad de entender y explicar los estados emocionales de otras personas, lo cual apoyaría anteriores hallazgos (Lockwood, Bird, Bridge y Viding, 2013; Samson Huber, y Gross, 2012; Schwenck et al., 2012).

De igual manera, este estudio sugiere que la ToM es el centro del déficit social en sujetos con autismo, porque enlaza tanto la atención conjunta como el reconocimiento de las emociones, y las subsecuentes habilidades como la creación de relaciones y la posterior inclusión social (Fletcher-Watson, McConnell, Manola y McConachie, 2014). Finalmente, Mazza et al. (2014) plantean que la empatía es un constructo multidimensional y que requiere de tres habilidades: el reconocimiento de emociones propias y de otros por expresiones faciales; compartir estados emocionales con otros, es decir, habilidad para experimentar emociones similares a los otros mientras se hacen conscientes de que es una simulación y no la propia emoción; y, por último, tomar la perspectiva de otra persona, aunque la distinción entre el yo y el otro permanezca intacta.

Por su parte, Grove et al. (2014) realizaron un estudio cuyo principal objetivo fue examinar la relación entre diferentes componentes de empatía entre individuos con vulnerabilidad genética al autismo. Para la medición de la empatía, los autores utilizaron el autorreporte Empathy Quotient - EQ (Baron-Cohen y Wheelwright, 2004) y para la teoria de la mente aplicaron Reading the Mind in the Eyes - RME (Baron-Cohen et al., 2001). Los resultados indicaron que los individuos con autismo tienen un deterioro en los cuatro factores latentes evaluados (empatía cognitiva, empatía afectiva, habilidades sociales y rendimiento basado en tareas), mientras que los padres presentaron dificultades intermedias. Este estudio concluye que las dificultades del procesamiento empático en sujetos con autismo no solamente están vinculadas con un procesamiento cognitivo atípico, sino que otras áreas pueden estar involucradas. 
Siguiendo con los estudios que muestran déficit en la empatía cognitiva, Pouw et al. (2013) examinaron, en niños autistas y en niños con desarrollo típico, la empatía afectiva y cognitiva, y su relación con la ToM. Para ello midieron la empatía con Empathy Questionnaire - EQ (Overgaauw, Güroğlu, Rieffe y Crone, 2014) y utilizaron dos tareas de falsa creencia (Baron-Cohen, Leslie y Frith, 1985) y videoclips cortos de Mr. Bean (personaje cómico). Los hallazgos muestran que los sujetos con autismo reportaron menos empatía cognitiva comparada con los del grupo control. Además, no se encontraron diferencias en los resultados de la empatía afectiva en comparación con el grupo control.

Con el objetivo de investigar el efecto de la sincronía interpersonal percibida cuando el sujeto (seguidor) ajusta sus movimientos para atraer los movimientos de la otra persona (líder)- sobre la empatía cognitiva y emocional en los individuos con autismo, Koehne et al. (2016) utilizaron el Movie for the assessment of social cognition MASC (Dziobek et al., 2006) y Reading the Mind in the Eyes task-RME (Baron-Cohen et al., 2001). Los resultados indicaron que los participantes generalmente reportan más empatía cognitiva con el compañero en condiciones de sincronía, que en las de asincronía. Se encontró una correlación positiva en el grupo de autistas entre la sincronía producida y la empatía cognitiva, como evalúa MASC y RME, lo cual muestra una alta sincronía producida asociada con altos niveles de empatía afectiva.

Por su parte, Mathersul et al. (2013) investigaron la comprensión en las inferencias conversacionales sutiles y la relación entre la ToM avanzada con la empatía cognitiva y afectiva. Para ello, utilizaron The Awareness of Social Inference Test - TASIT (McDonald, Flanagan, Martin y Saunders, 2004), Interpersonal Reactivity Index - IRI (Davis, 1980), Empathy Quotient - EQ (Baron-Cohen y Wheelwright, 2004). Los resultados mostraron que los adultos con autismo de alto funcionamiento son capaces de realizar juicios básicos de ToM. Sin embargo, carecen de interpretación de expresiones de sarcasmo y engaño, que requieren una ToM más compleja. El estudio también sugiere que los autistas tienen un déficit particular en reconocer e interpretar creencias e intenciones (características asignadas a la ToM), así como el significado de las interacciones indirectas. De otro lado, la empatía afectiva no estuvo relacionada significativamente con la ToM avanzada, esto sugiere que la empatía y la ToM afectiva son procesos diferentes, y apoya planteamientos previos que sustentan que la empatía afectiva implica la capacidad de responder emocionalmente ante el evento social presentado; lo cual no necesariamente involucra la capacidad de inferir el estado emocional de los otros (Decety, 2010; Rankin, Kramer y Miller, 2005).

Un estudio reciente de Peterson (2014) que explora las diferencias individuales en la comprensión de la ToM y su relación con la empatía en niños autistas, utilizó la prueba de falsa creencia Sally-Anne Task (Baron-Cohen et al., 1985) Misleading 
Contents False Belief Task - MCFB (Wellman y Liu, 2004). Los resultados muestran que la comprensión de la ToM no está relacionada con la empatía en los niños con autismo; también que, aunque los niños de mayor edad reflejan mayor rendimiento en pruebas de falsa creencia, son menos empáticos que los más jóvenes con desarrollo típico, que tienen un rendimiento más bajo en falsa creencia.

Según estos estudios empíricos, existen diferentes instrumentos para la medición de la empatía y la ToM. No obstante, los más utilizados son: Reading the Mind in the Eyes Test - RME (Baron-Cohen et al., 2001), Interpersonal Reactivity Index - IRI (Davis, 1980), Empathy Quotient - EQ (Baron-Cohen y Wheelwright, 2004), y tareas de falsa creencia como Sally-Anne Task (Baron-Cohen et al., 1985). Estos instrumentos utilizados muestran que el posible déficit de los autistas para los comportamientos empáticos y sociales se sustenta en su dificultad para comprender y explicar los estados mentales propios y ajenos. Aunque los estímulos con valencia positiva suelen ser mejor correspondidos que los estados emocionales o pensamientos negativos, no logran realizar ningún tipo de interpretación cuando solamente observan la región de los ojos. También muestran déficits en habilidades sociales y empatía cognitiva y afectiva, aunque esta última no suele encontrarse relacionada con las habilidades de la ToM, contrario de la empatía cognitiva que suele vincularse con ella.

\section{Circuitos cerebrales en autismo}

Schulte-Rüther et al. (2014) estudiaron con fMRI los mecanismos neurales que subyacen a la empatía, utilizando tareas que requerían evaluar la perspectiva individual (SelfTask) y social (Other Task) para una adecuada construcción del contexto interpersonal, con la Empathizing Task - ET (Schulte-Rüther y Greimel, 2011). Los resultados mostraron diferencias de acuerdo a la edad de los sujetos, en la realización de tareas relacionadas con el auto-reconocimiento. Se evidenció disminución de la actividad en el hemisferio derecho de la corteza prefrontal dorsolateral, la ínsula anterior, la corteza prefrontal inferior, la zona medial de la corteza prefrontal, y la corteza occipital. Estos hallazgos sugieren que los sujetos con autismo pueden ser capaces de identificar los estados emocionales de los otros, aunque pueden presentar un déficit en la evaluación de una reacción emocional propia. El estudio muestra que el refinamiento de las redes cerebrales funcionales relacionadas con habilidades sociales y cognitivas en autistas continúa en la edad adulta, sugiriendo que durante el curso del desarrollo, individuos con autismo pueden ser capaces de adquirir una estrategia cognitiva para acceder a las emociones de otras personas. 
Así mismo, Fan et al. (2014), al examinar con fMRI el procesamiento empático y la comprensión social del dolor, encontraron que los sujetos con autismo tuvieron una activación en el giro precentral izquierdo, el giro temporal inferior derecho y el giro lingual izquierdo en la percepción de situaciones dolorosas. De igual forma, se evidenció que hubo un mayor cambio en el grupo control que en los autistas en la señal de la corteza cingulada anterior media (CCAm) y la corteza insular anterior (CIA). Los sujetos con autismo presentaron una mayor señal hemodinámica en las cortezas somatosensoriales y en el giro frontal inferior en respuesta a la percepción del dolor de los otros. En tanto a la comprensión social del estímulo, el grupo de autistas, en relación al grupo control, no tuvo una activación significativa en la corteza prefrontal medial (CPF) derecha, la corteza cingulada posterior bilateral, el surco temporal superior (STS) y la unión temporoparietal (UTP). Los resultados muestran que los participantes con autismo conservaron una típica resonancia del área sensoriomotora y que los umbrales más bajos de dolor se acoplaron con rasgos autistas más reducidos, sugiriendo un procesamiento sensorial atípico en autistas que influye en el procesamiento empático. Estos resultados sugieren que, además de la reducción de los umbrales de dolor, las personas con autismo muestran una excitación acentuada empática, aunque la comprensión social del dolor sea deteriorada al percibir la angustia de los otros.

En esta misma línea, Greimel et al. (2010) utilizaron fMRI y una prueba que contiene rostros que expresan felicidad, tristeza y neutralidad, para mostrar los mecanismos cerebrales de la empatía en el autismo y para explorar las contribuciones que el vínculo familiar tiene con la empatía. Los resultados mostraron que el grupo con autismo tenía una disminución en la activación del giro fusiforme en comparación con el grupo control. Por su parte, los padres de los sujetos autistas también mostraron activación reducida del giro fusiforme cuando inferían las emociones de los otros. Los autores concluyen que las anomalías compartidas en la activación del giro fusiforme, tanto en adolescentes como en parientes de primer grado, constituyen una desviación fundamental en autismo; brindando evidencia de que los mecanismos de imitación están implicados en el déficit para establecer relaciones empáticas en autismo.

De otro lado, Hoffmann et al. (2016) utilizaron fMRI en estado de reposo con el objetivo de estudiar si los pacientes con autismo mostraban, durante la empatía, dificultades en la distinción de sí mismos y de los otros. Los resultados mostraron que el grupo de autismo califican las emociones negativas y positivas con menor intensidad para sí mismos y los otros en comparación al grupo control. También encontraron que los autistas tuvieron significativamente menores puntajes, lo cual se relaciona negativamente con la severidad del síntoma. En cuanto al análisis de neuroimagen, los autores exponen que el giro supramarginal derecho mostró mar- 
cada conectividad en patrones relativos a la unión temporoparietal (UTP) derecha y al giro supramarginal izquierdo (GSM), al igual que la corteza cingulada media (CCm) y la corteza prefrontal lateral dorsal. En contraste, la UTP derecha mostró una fuerte conexión comparada con el GSM derecho y la corteza prefrontal medial. También, los sujetos autistas mostraron una conectividad funcional reducida de UTP derecha a UTP izquierda en el precúneo, la corteza cingulada posterior y la corteza prefrontal medial. Los autores concluyen que los sujetos con autismo presentaron déficits en el procesamiento de la teoría de mente. Finalmente, los autistas no son más propensos a proyectar sus sentimientos hacia los demás ni a percibir y comprender los sentimientos de los demás con sus propios sentimientos.

En un estudio de revisión realizado por Thioux y Keysers (2010) se expone una mayor actividad en la ínsula y la corteza cingulada anterior en las personas empáticas testigos de disgustos faciales o al darse cuenta de que su pareja está experimentando dolor. Estos resultados indican que los circuitos compartidos pueden ser la clave de la cognición social, proporcionando una perspectiva vicaria de los sentimientos de los otros. De igual forma, sugieren que los sujetos autistas no presentan dificultades con la imitación de las acciones dirigidas a un objetivo. Una diferencia importante es que los niños autistas tienden a presentar una menor activación en la corteza premotora que sus pares, específicamente en la parte ventral, contrastando resultados en población autista adulta, en donde no se encuentra diferencia en esta activación en relación a los adultos con desarrollo típico.

Esta conclusión encuentra soporte en un reciente estudio (Beall, Moody, McIntosh, Hepburn y Reed, 2008) que mostró que el mimetismo facial mejora con la edad, puesto que niños mayores presentan más ocurrencias de respuestas musculares congruentes con rostros felices. Una revisión reciente muestra el rol de la ínsula en la empatía, Tousignant et al. (2016) exponen que la observación de otro mostrando el rostro o el cuerpo en sufrimiento, comúnmente indican una activación en la ínsula anterior bilateral (IAb), el giro frontal inferior adyacente (GFIa), la corteza cingulada medial anterior y anterior dorsal; señalando que estas áreas están implicadas en los sentimientos hacia otros y los comportamientos empáticos. También se expone que las bases neurales implicadas en la distinción del yo y el otro, que involucran un sentimiento de autoconciencia, están asociadas a regiones como la unión temporoparietal (UTP) y el lóbulo parietal inferior (LPi).

La vinculación de técnicas de neuroimagen al estudio de pacientes autistas ha sido un avance importante, puesto que posibilitan el análisis de las redes neurales que subyacen al funcionamiento social deficiente observado en esta población. Como pudo apreciarse en este apartado, existe evidencia de regiones cerebrales implicadas en el procesamiento de las emociones y en la interpretación de los estímulos. En 
este sentido, las estructuras cerebrales reportadas en los estudios como relacionadas con la empatía son: la corteza prefrontal, específicamente la parte derecha, la cual orquesta los circuitos principales de las funciones ejecutivas que posibilitan acciones de regulación conductual dirigidas a una acción (García-Molina, Enseñat-Cantallops, Tirapu-Ustárroz y Roig-Rovira, 2009); la corteza anterior, la cual permite procesos de autoconciencia; el giro fusiforme, implicado en el reconocimiento de rostros; y la unión temporoparietal, implicada en la regulación de las emociones (Fan et al., 2014; Greimel et al., 2010).

\section{Conclusiones y discusión}

Esta revisión tuvo como objetivo principal mostrar cómo se ha estudiado el déficit de la empatía en sujetos con autismo desde tres elementos básicos: el concepto de empatía, la medición y las áreas cerebrales involucradas. Se encontró que en los diferentes conceptos de empatía suele existir una división en sus mecanismos: el procesamiento afectivo, concerniente a los estímulos que desencadenan reacciones emocionales; y el procesamiento cognitivo, el cual se refiere a la habilidad para comprender pensamientos de los otros y está estrechamente relacionado con la ToM (Cuff, Brown, Taylor y Howat, 2014). Los estudios sobre la empatía afectiva han mostrado resultados diversos, por un lado, muestran que solamente cuando la valencia del estímulo es positiva los sujetos son capaces de corresponderse emocionalmente con otra persona (Mazza et al., 2014). Contrario a esto, estudios sobre el sufrimiento y el dolor plantean que los sujetos con autismo pueden presentar angustia y sentimientos similares al que está recibiendo el estímulo doloroso, aunque no puedan comprender qué está pasando (Fan et al., 2014; Tousignant et al., 2016) y, más aún, si es la pareja o una persona conocida (Thioux y Keysers, 2010). Esto muestra que los sujetos con autismo logran sentir lo que pasa a su alrededor, sugiriendo que no están enajenados totalmente de las claves sociales y que son capaces de corresponderse con los otros en situaciones con contenido emocional.

Los estudios realizados en torno a la empatía cognitiva han mostrado que existe un déficit en el procesamiento y reconocimiento de estados mentales, sentimientos y pensamientos propios y ajenos (Pasalich et al., 2014), evidenciando que la dificultad en esta dimensión podría atribuirse al marcado déficit en la habilidad de entender y explicar los estados emocionales de las otras personas (Hirvelä y Helkama, 2011; Jones et al., 2010; Lockwood et al., 2013); es decir, en la capacidad de crear una teoría de la mente, la cual se ha visto notablemente deteriorada en los niños con autismo (Pouw et al., 2013). Con base en esto, se plantea que los estudios sobre los procesos 
de empatía cognitiva y afectiva han encontrado que los adultos con autismo tienen impedimentos en el proceso mental de tomar la perspectiva de los otros, pero no en la preocupación empática, la cual les permite sentir filiación emocional hacia las otras personas, aun cuando no comprenden lo que sucede (Dziobek et al., 2008). Lo anterior es respaldado por estudios que sugieren que, aunque el procesamiento cognitivo esté deteriorado, el procesamiento emocional se encuentra intacto (Dziobek et al., 2008; Jones et al., 2010; Mazza et al., 2014). Aunque existe esta evidencia, los estudios no logran converger en definiciones más estandarizadas, debido en parte a la dificultad planteada sobre la definición del concepto de empatía.

Respecto a la medición de la empatía en autismo, los estudios revisados muestran el uso de diferentes instrumentos. Se observó que los instrumentos más utilizados en las investigaciones han sido los autorreportes y cuestionarios como el Índice de Reactividad Interpersonal - IRI (Davis, 1980), Empathy Quotient - EQ (Baron-Cohen y Wheelwright, 2004) y el Multifaceted Empathy Test - MET (Dziobek et al., 2008), que se han empleado para medir los componentes afectivos y cognitivos de la empatía. De igual forma, se han utilizado tareas con el fin de evaluar ToM y su relación con la empatía, por ejemplo las pruebas de falsa creencia como la Sally-Anne Task (Baron-Cohen et al., 1985) y Misleading Contents False Belief Task - MCFB (Wellman y Liu, 2004), y las pruebas para el reconocimiento de emociones básicas, como The Awareness of Social Inference Test - TASIT (McDonald et al., 2004). Esto indica que la medición de la empatía no está claramente definida y se tiende a utilizar pruebas típicamente empleadas para medición de procesos como procesamiento de emociones y ToM. Lo anterior apoya la premisa según la cual las dificultades en la delimitación conceptual en la empatía afectan la validez de las mediciones.

De otro lado, aunque no existe una delimitación conceptual única, los estudios en neuroimágenes muestran más coherencia en sus resultados. Las evidencias son consistentes al mostrar áreas que presentan funcionamiento atípico, como la corteza prefrontal (Fan et al., 2014; Schulte-Rüther et al., 2014), la corteza insular (Bird et al., 2010; Fan et al., 2014), la unión temporoparietal (Fan et al., 2014; Tousignant et al., 2016) y el giro fusiforme (Greimel et al., 2010). Estas áreas posibilitan el reconocimiento de rostros, la capacidad para la imitación y procesamientos que favorecen el comportamiento social y adaptativo. Otro punto importante es que se ha demostrado que los sujetos con autismo tienen un procesamiento atípico de su red especular, la cual posibilita la comprensión de los actos ajenos, incluyendo los sentimientos y las emociones, que responden a las intenciones y movimientos de los otros y posibilita su comprensión (Moya-Albiol et al., 2010).

La presente revisión permite inferir la complejidad en la estrecha relación entre las dificultades en el procesamiento empático y el déficit social encontrado en sujetos 
con autismo. Se considera importante detenerse en los hallazgos sobre el procesamiento de la empatía afectiva, que permite sentir correspondencia emocional con otro. Esto es especialmente importante porque: 1) es punto de partida para el planteamiento de intervenciones que incluyan el manejo de emociones y de la empatía; y 2) permite estudiar el funcionamiento afectivo de la empatía a partir de diferentes contextos sociales. A nivel teórico y experimental, se consideran dos elementos relevantes a tener en cuenta en las futuras investigaciones: 1) delimitar muy bien qué se entiende por empatía y, con ello, no perder la validez de las mediciones en los estudios (Cuff et al., 2014); y 2) lograr un mayor nivel de naturalismo en la medición de procesos complejos de la cognición social como la empatía es uno de los mayores retos para contribuir a métodos y técnicas de medición más ecológicas (Herrera, 2018). Quizá todo esto permita encontrar otras diferencias en el procesamiento, que permitan avanzar más en el conocimiento y la comprensión del sujeto autista.

\section{Referencias}

AAN HET ROT, M., y HOGENELST, K. (2014). The influence of affective empathy and autism spectrum traits on empathic accuracy. PLoS One, 9(6), 241-249.

ALBIERO, P., MATRICARDI, G., SPELTRI, D., y TOSO, D. (2009). The assessment of empathy in adolescence: A contribution to the Italian validation of the «Basic Empathy Scale». Journal of adolescence, 32(2), 393-408.

BARNETT, G., y MANN, R. E. (2013). Empathy deficits and sexual offending: A model of obstacles to empathy. Aggression and violent behavior, 18(2), 228-239.

BARON-COHEN, S., LESLIE, A. M. y FRITH, U. (1985). Does the autistic child have a «theory of mind»? Cognition, 21(1), 37-46.

BARON-COHEN, S. y WHEELWRIGHT, S. (2004). The empathy quotient: an investigation of adults with Asperger syndrome or high functioning autism, and normal sex differences. Journal of autism and developmental disorders, 34(2), 163-175.

BARON-COHEN, S., WHEELWRIGHT, S., SKINNER, R., MARTIN, J. y CLUBLEY, E. (2001). The autism-spectrum quotient (AQ): Evidence from asperger syndrome/high-functioning autism, malesand females, scientists and mathematicians. Journal of autism and developmental disorders, 31(1), 5-17.

BEALL, P. M., MOODY, E. J., MCINTOSH, D. N., HEPBURN, S. L. y REED, C. L. (2008). Rapid facial reactions to emotional facial expressions in typically developing children and children with autism spectrum disorder. Journal of experimental child psychology, 101(3), 206-223. 
BIRD, G., SILANI, G., BRINDLEY, R., WHITE, S., FRITH, U. y SINGER, T. (2010). Empathic brain responses in insula are modulated by levels of alexithymia but not autism. Brain, 133(5), 1515-1525.

BIRD, G. y VIDING, E. (2014). The self to other model of empathy: providing a new framework for understanding empathy impairments in psychopathy, autism, and alexithymia. Neurosciencey biobehavioral reviews, 47, 520-532.

BLAIR, R. J. R. (2005). Responding to the emotions of others: dissociating forms of empathy through the study of typical and psychiatric populations. Consciousness and cognition, 14(4), 698-718.

BOS, J. y STOKES, M. A. (2018). Cognitive empathy moderates the relationship between affective empathy and wellbeing in adolescents with autism spectrum disorder. European Journal of Developmental Psychology, 1-14. DOI: 10.1080/17405629.2018.1444987

CATTANEO, L. y RIZZOLATTI, G. (2009). The mirror neuron system. Archives of Neurology, 66(5), 557-56o.

CLARK, A. J. (2010). Empathy and sympathy: Therapeutic distinctions in counseling. Journal of mental health counseling, 32(2), 95.

COOK, R., BREWER, R., SHAH, P. y BIRD, G. (2013). Alexithymia, not autism, predicts poor recognition of emotional facial expressions. Psychological Science, 24(5), 723-732.

CUFF, B. M., BROWN, S. J., TAYLOR, L. y HOWAT, D. J. (2014). Empathy: a review of the concept. Emotion Review, 8(2), 144-153.

DADDS, M. R., HUNTER, K., HAWES, D. J., FROST, A. D., VASSALlO, S., BUNN, P. [...] y EL MASRY, Y. (2008). A measure of cognitive and affective empathy in children using parent ratings. Child psychiatry and human development, 39(2), 111-122.

DAVIS, M. H. (1980). A multidimensional approach to individual differences in empathy. Journal of Personality and Social Psychology, 10, 1-85.

DECETY, J. (2010). The neurodevelopment of empathy in humans. Developmental neuroscience, $32(4), 257-267$.

DECETY, J. y JACKSON, P. L. (2006). A social-neuroscience perspective on empathy. Current Directions in Psychological Science, 15(2), 54-58.

DECETY, J. y LAMM, C. (2006). Human empathy through the lens of social neuroscience. The Scientific World Journal, 6, 1146-1163.

DECETY, J. y MEYER, M. (2008). From emotion resonance to empathic understanding: A social developmental neuroscience account. Development and Psychopathology, 20(4), 1053-1080.

DECETY, J. y MICHALSKA, K. J. (2010). Neurodevelopmental changes in the circuits underlying empathy and sympathy from childhood to adulthood. Developmental science, 13(6), 886-899. 
DZIOBEK, I., FLECK, S., KALBE, E., ROGERS, K., HASSENSTAB, J., BRAND, M. [...] y CONVIT, A. (2006). Introducing MASC: a movie for the assessment of social cognition. Journal of autism and developmental disorders, 36(5), 623-636.

DZIOBEK, I., ROGERS, K., FLECK, S., BAHNEMANN, M., HEEKEREN, H. R., WOLF, O. T. y CONVIT, A. (2008). Dissociation of cognitive and emotional empathy in adults with Asperger syndrome using the Multifaceted Empathy Test (MET). Journal of autism and developmental disorders, 38(3), 464-473.

FAN, Y. T., CHEN, C., CHEN, S. C., DECETY, J. y CHENG, Y. (2014). Empathic arousal and social understanding in individuals with autism: evidence from fMRI and ERP measurements. Social cognitive and affective neuroscience, 9(8), 1203-1213.

FERNÁNDEZ-PINTO, I., LÓPEZ-PÉREZ, B.y MÁRQUEZ, M. (2008). Empatía: Medidas, teorías y aplicaciones en revisión. Anales de psicología, 24(2), 284-298.

FESHBACH, N. D. (1975). Empathy in children: Some theoretical and empirical considerations. The Counseling Psychologist, 5(2), 25-30.

FLETCHER-WATSON, S., MCCONNELL, F., MANOLA, E. y MCCONACHIE, H. (2014). Interventions based on the Theory of Mind cognitive model for autism spectrum disorder (ASD). Cochrane Database of Systematic Reviews, 3, 1-82.

GARCÍA-MOLINA, A., ENSEÑAT-CANTALLOPS, A., TIRAPU-USTÁRROZ, J. y ROIG-ROVIRA, T. (2009). Maduración de la corteza prefrontal y desarrollo de las funciones ejecutivas durante los primeros cinco años de vida. Revista de neurología, 48(8), 435-440.

GEDAY, J., GJEDDE, A., BOLDSEN, A.-S. y KUPERS, R. (2003). Emotional valence modulates activity in the posterior fusiform gyrus and inferior medial prefrontal cortex in social perception. Neuroimage, $18(3), 675-684$.

GEER, J. H., ESTUPINAN, L. A. y MANGUNO-MIRE, G. M. (200o). Empathy, social skills, and other relevant cognitive processes in rapists and child molesters. Aggression and violent behavior, $5(1), 99-126$.

GOLDMAN, A. I. (1993). Ethics and cognitive science. Ethics, 103(2), 337-360.

GREIMEL, E., SCHULTE-RÜTHER, M., KIRCHER, T., KAMP-BECKER, I., REMSCHMIDT, H., FINK, G. R. [...] y KONRAD, K. (2010). Neural mechanisms of empathy in adolescents with autism spectrum disorder and their fathers. Neuroimage, 49(1), 1055-1065.

GROVE, R., BAILliE, A., ALLISON, C., BARON-COHEN, S. y HOEKSTRA, R. A. (2014). The latent structure of cognitive and emotional empathy in individuals with autism, first-degree relatives and typical individuals. Molecular autism, $5(1), 1$.

HAGENMULLER, F., ROESSLER, W., WITTWER, A. y HAKER, H. (2014). Empathic resonance in Asperger syndrome. Research in Autism Spectrum Disorders, 8(7), 851-859. 
HAPPÉ, F. G. (1994). An advanced test of theory of mind: Understanding of story characters' thoughts and feelings by able autistic, mentally handicapped, and normal children and adults. Journal of autism and developmental disorders, 24(2), 129-154.

HERRERA, E. (2018). Enfoques de la medición de la emoción y la empatía en desordenes de conducta. En: Bravo, O. (Ed.) Una década del programa de Psicología en la Universidad Icesi. Trayectorias y enfoques plurales. Cali, Colombia: Editorial Universidad Icesi.

HIRVELÄ, S. y HELKAMA, K. (2011). Empathy, values, morality and Asperger's syndrome. Scandinavian journal of psychology, 52(6), 560-572.

HOFFMANN, F., KOEHNE, S., STEINBEIS, N., DZIOBEK, I. y SINGER, T. (2016). Preserved selfother distinction during empathy in autism is linked to network integrity of right supramarginal gyrus. Journal of autism and developmental disorders, 46(2), 637-648.

JONES, A. P., HAPPÉ, F. G., GILBERT, F., BURNETT, S. y VIDING, E. (2010). Feeling, caring, knowing: different types of empathy deficit in boys with psychopathic tendencies and autism spectrum disorder. Journal of Child Psychology and Psychiatry, 51(11), 1188-1197.

KOEHNE, S., HATRI, A., CACIOPPO, J. T. y DZIOBEK, I. (2016). Perceived interpersonal synchrony increases empathy: Insights from autism spectrum disorder. Cognition, 146, 8-15.

LANG, P. , BRADLEY, M., y CUTHBERT, B.(1997). International Affective Picture System (IAPS): Technical manual and affective ratings. Gainesville, FL: University of Florida.

LÁZARO, J. C. F. y SOLÍS, F. O. (2008). Neuropsicología de lóbulos frontales, funciones ejecutivas y conducta humana. Revista Neuropsicología, Neuropsiquiatría y Neurociencias, 8(1), 47-58.

LOCKWOOD, P. L. (2016). The anatomy of empathy:Vicarious experience and disorders of social cognition. Behavioural brain research, 311, 255-266.

LOCKWOOD, P. L., BIRD, G., BRIDGE, M. y VIDING, E. (2013). Dissecting empathy: high levels of psychopathic and autistic traits are characterized by difficulties in different social information processing domains. Frontiers in human neuroscience, 7, 760.

MATHERSUL, D., MCDONALD, S. y RUSHBY, J. A. (2013). Understanding advanced theory of mind and empathy in high-functioning adults with autism spectrum disorder. Journal of clinical and experimental neuropsychology, 35(6), 655-668.

MAZZA, M., PINO, M. C., MARIANO, M., TEMPESTA, D., FERRARA, M., DE BERARDIS, D. [...] y VALENTI, M. (2014). Affective and cognitive empathy in adolescents with autism spectrum disorder. Frontiers in human neuroscience, 8, 791.

MCDONALD, S., FLANAGAN, S., MARTIN, I. y SAUNDERS, C. (2004). The ecological validity of TASIT: A test of social perception. Neuropsychological Rehabilitation, 14(3), 285-302. 
MEHRABIAN, A. (1996). The Balanced Emotional Empathy Scale (BEES). Documento inédito disponible en: 1130 Alta Mesa Road, Monterey, CA, USA 93940.

MOYA-ALBIOL, L., HERRERO, N. y BERNAL, M. C. (2010). Bases neuronales de la empatía. Rev Neurol, 5o(2), 89-100.

OLIVEIRA-SILVA, P. y GONÇALVES, Ó. F. (2011). Responding empathically: A question of heart, not a question of skin. Applied psychophysiology and biofeedback, 36(3), 201-207.

OVERGAAUW, S., GÜROĞLU, B., RIEFFE, C. y CRONE, E. A. (2014). Behavior and Neural Correlates of Empathy in Adolescents. Developmental neuroscience, 36(3-4), 210-219.

PASALICH, D. S., DADDS, M. R. y HAWES, D. J. (2014). Cognitive and affective empathy in children with conduct problems: Additive and interactive effects of callous-unemotional traits and autism spectrum disorders symptoms. Psychiatry research, 219(3), 625-630.

PELLIGRA, V. (2011). Empathy, guilt-aversion, and patterns of reciprocity. Journal of Neuroscience, Psychology, and Economics, 4(3), 161.

PETERSON, C. (2014). Theory of mind understanding and empathic behavior in children with autism spectrum disorders. International Journal of Developmental Neuroscience, 39, 16-21.

POUW, L. B., RIEFFE, C., OOSTERVELD, P., HUSKENS, B. y STOCKMANN, L. (2013). Reactive/proactive aggression and affective/cognitive empathy in children with ASD. Research in developmental disabilities, 34(4), 1256-1266.

RANKIN, K. P., KRAMER, J. H. y MILLER, B. L. (2005). Patterns of cognitive and emotional empathy in frontotemporal lobar degeneration. Cognitive and Behavioral Neurology, 18(1), 28-36.

RUGGIERI, V. L. (2013). Empatía, cognición social y trastornos del espectro autista. Rev Neurol, 56(Supl 1), S13-21.

SAMSON, A. C., HUBER, O. y GROSS, J. J. (2012). Emotion regulation in Asperger's syndrome and high-functioning autism. Emotion, 12(4), 659.

SCHULTE-RÜTHER, M. y GREIMEL, E. (2011). Brain networks supporting empathy. From DNA to Social Cognition, 47-61.

SCHULTE-RÜTHER, M., GREIMEL, E., PIEFKE, M., KAMP-BECKER, I., REMSCHMIDT, H., FINK, G. R. [...] y KONRAD, K. (2014). Age-dependent changes in the neural substrates of empathy in autism spectrum disorder. Social cognitive and affective neuroscience, 9 (8), 1118-1126.

SCHWENCK, C., MERGENTHALER, J., KELLER, K., ZECH, J., SALEHI, S., TAURINES, R. [...] y WARNKE, A. (2012). Empathy in children with autism and conduct disorder: Group-specific profiles and developmental aspects. Journal of Child Psychology and Psychiatry, 53 (6), 651-659. 
SHAMAY-TSOORY, S., TOMER, R., YANIV, S. y AHARON-PERETZ, J. (2002). Empathy deficits in Asperger syndrome: A cognitive profile. Neurocase, 8(2), 245-252.

SINGER, T. y LAMM, C. (2009). The social neuroscience of empathy. Annals of the New York Academy of Sciences, 1156(1), 81-96.

STOCKS, E. L., LISHNER, D. A., WAITS, B. L. y DOWNUM, E. M. (2011). I'm embarrassed for you: The effect of valuing and perspective taking on empathic embarrassment and empathic concern. Journal of Applied Social Psychology, 41(1), 1-26.

STOTLAND, E. (1969). Exploratory investigations of empathy. Advances in experimental social psychology, 4, 271-314.

SUCKSMITH, E., ALLISON, C., BARON-COHEN, S., CHAKRABARTI, B. y HOEKSTRA, R. (2013). Empathy and emotion recognition in people with autism, first-degree relatives, and controls. Neuropsychologia, 51(1), 98-105.

TITCHENER, E. (1909). Lectures on the Experimental Psychology of the Thought-Processes. New York: Macmillan.

THIOUX, M. y KEYSERS, C. (2010). Empathy: shared circuits and their dysfunctions. Dialogues in clinical neuroscience, 12(4), 546-552.

TOUSIGNANT, B., EUGÈNE, F. y JACKSON, P. L. (2017). A developmental perspective on the neural bases of human empathy. Infant Behavior and Development, 48(A), 5-12.

WELLMAN, H. M. y LIU, D. (2004). Scaling of theory of mind tasks. Child development, 75 (2), 523-541.

ZAHAVI, D. (2008). Simulation, projection and empathy. Consciousness and cognition, 17(2), 514-522.

ZAKI J., BOLGER N. y OCHSNER K. (2008). It takes two: the interpersonal nature ofempathic accuracy. Psychological Science, (19), 399-404. 